\title{
WHAT IS A THERAPEUTIC POTENTIAL OF N-ACETYLCYSTEINE IN LUNG SILICOSIS?
}

\author{
ADAMCAKOVA JANA, MOKRA DANIELA
}

Department of Physiology, Jessenius Faculty of Medicine in Martin, Comenius University in Bratislava, Martin, Slovakia

\begin{abstract}
A b s tract
Lung silicosis is a serious pulmonary disease caused by an exposure of lung to inhaled silicon dioxide $\left(\mathrm{SiO}_{2}\right)$ or silica. Although pathomechanisms of the disease have not been fully elucidated, oxidative stress has been recognized as a fundamental factor triggering a fibrotizing inflammation leading to irreversible changes in lung tissue. Based on this knowledge, therapeutic potential of various antioxidants has been intensively discussed. Among them, N-acetylcysteine with its multiple anti-inflammatory and antioxidant actions and a long-term experience with its clinical use in various diseases appears as a very promising choice. The purpose of this article is to review the therapeutic effects of $\mathrm{N}$-acetylcysteine particularly in relation to a lung injury and to point out a potential of $\mathrm{N}$-acetylcysteine in the treatment of lung silicosis.
\end{abstract}

Key words: silica, lung silicosis, oxidative stress, $\mathrm{N}$-acetylcysteine, antioxidants

\section{INTRODUCTION}

Lung silicosis is a form of interstitial lung fibrosis (pneumoconiosis) which may be potentially fatal or may lead to irreversible fibrotic changes. Lung silicosis is caused by an intensive and/or a long-term exposure of lung to inhaled silicon dioxide $\left(\mathrm{SiO}_{2}\right)$ or silica, especially to particles smaller than $10 \mu \mathrm{m}$ in diameter, released from stones or sand in mining and industrial processing. Therefore, silicosis is a typical occupational disease in miners and stonemasons (1). While the development of lung silicosis is conditioned by a cumulative dose of silica exposure, there have been several forms of acute and chronic silicosis classified according to duration and intensity of exposure to respirable silica dust $(2,3)$. Despite lung silicosis is an old disease, there is no causal treatment. Patients are treated symptomatically using bronchodilators, antitusics, and mucolytics and an avoiding of further exposures to particulate silica is recommended. As the last therapeutic possibility after the exhaustion of other options, a lung transplantation may be suggested, however, a long-term survival after the lung transplantation is poor (3). Although pathomechanisms of the disease have not been fully elucidated and a complex action of several factors is proposed, oxidative stress has been recognized as a key factor triggering inflammation and fibrosis, finally leading to irreversible changes in the lung (4). An increasing number of studies has revealed a therapeutic potential of various herbal compounds and "classical" antioxidants. Among them, N-acetylcysteine with its multiple anti-inflammatory and antioxidant actions and a long-term experience with its wide clinical use appears as a valuable choice (5). The purpose of this article is, firstly, to provide a short review of the role of oxidative stress in the patho-

Corresponding author: Daniela Mokra, Prof. MD., PhD.; e-mail: daniela.mokra@uniba.sk

(C) 2021 Mokra D. et al.

This work is licensed under the Creative Commons Attribution-NonCommercial-NoDerivs 4.0 License (https://creativecommons.org/licenses/by-nc-nd/4.0/) 
physiology of silicosis. In addition, the article points out therapeutic effects of $\mathrm{N}$-acetylcysteine (NAC) particularly in relation to a lung injury and presents possible benefits of NAC in the treatment of lung silicosis.

\section{Oxidative stress in the pathophysiology of lung silicosis}

The oxidative stress and oxidative stress-related changes are fundamental in the development of lung silicosis. Large quantities of reactive oxygen species (ROS) are formed already in the direct contact with silica crystals, particularly with those freshly cut, because of piezoelectric properties of silica crystals (6). Inhaled silica particles are rapidly recognized by the surface receptors of alveolar macrophages (AM) and engulfed by these cells. However, the system of lysosomes is not able to breakdown this type of particles, which results into a damage of lysosomal membrane and a discharge of lysosomal enzymes in the tissue. The released silica is subsequently engulfed by other AM and the process continues in a vitious cycle (7).

The released lysosomal enzymes, high concentrations of generated ROS, and activation of surface receptors on the immune cells stimulate pro-inflammatory pathways including nuclear factor (NF)-kappa (к)B contributing to the activation of NLRP3 inflammasome with subsequent mitochondrial or cell apoptosis, pyroptosis, a highly pro-inflammatory type of cell death, and further generation of inflammation (4). Inflammation is associated with a production of additional quantities of ROS in the activated inflammatory cells which in turn stimulate the inflammation (8-10). The situation is further complicated with a silica-induced degradation of some antioxidant systems, e.g. peroxiredoxin (11). The perpetuation of this harmful process leads not only to a chronic lung inflammation but pro-inflammatory cytokines, e.g. interleukins (IL)- 1 and IL-18, and tumor necrosis factor (TNF) $\alpha$, stimulate the recruitment and proliferation of fibroblasts and mesenchymal cells to form fibroblastic foci and to produce the components of extracellular matrix including collagen, which results into an irreversible fibrotic scarring of the lung (12).

\section{Antioxidants in the lung silicosis}

Considering the role of excessive concentrations of ROS in the progression of silica-induced lung inflammation and fibrosis, the administration of antioxidants may be of benefit. In addition to the mitigation of oxidative stress, some antioxidants have a potential to influence cell apoptosis and/or to enhance autophagy, a special form of tissue-friendly elimination of damaged cells, toxic macromolecules, and injured organelles (13-15). While the potential of some phytomedicines with antioxidant actions has been recently published by us elsewhere (16), this article provides a review of therapeutic effects of NAC, a well-known and widely used antioxidant, in the lung injury generally and points out its possible benefits in the lung silicosis.

\section{Effects of NAC}

NAC is N-acetyl derivative of amino acid L-cysteine. Thanks to a content of thiol (-SH) group, NAC works as a direct scavenger of ROS, e.g. of hydrogen peroxide, hydroxyl radicals, and hypochlorous acid, or nitrogen dioxide, and inhibits the activity of cyclooxygenase-2 and membrane lipid peroxidation induced by inflammation (17). NAC is rapidly deacetylated to cysteine, a precursor of glutathione (GSH) synthesis in cells, and thereafter it regulates an intracellular redox status. NAC binds transition metal ions and heavy metal ions, forming complexes which are readily excreted from the body (18). NAC also exerts some anti-inflammatory and lung-protective actions as it mitigates a generation of pro-inflammatory cytokines TNF $\alpha$, IL-1 $\beta$ and IL- 6 by suppressing the activity of NF- $\kappa B$ (19). Besides potent antioxidant and anti-inflammatory effects, NAC reduces the viscosity and elasticity of mucus because of its ability to split disulphide bonds in high-molecular-weight glycoproteins in mucus (18), which is useful e.g. in cystic fibrosis. In addition to many positive pharmacological properties, a wide use of this drug is also related to its high stability and low price. Because of low bioavailability, NAC has a negligible toxicity. In standard dosing 
with a maximum licensed oral dose for a chronic use of $600 \mathrm{mg} / \mathrm{day}$, the treatment with NAC is well tolerated (20). Analyses of occurence of adverse effects in NAC-treated patients with chronic respiratory diseases showed no increase in the frequency of side effects compared to placebo-treated groups $(21,22)$. The side effects of oral NAC, such as nausea, vomiting, or diarrhea are rare and limited to very high concentrations (20). For instance, the administration of oral NAC for 22 weeks in a doubled dose than the maximum recommended (1,200 mg/day) in patients with chronic bronchitis caused only in one patient of 78 in total the adverse clinical symptoms such as dizziness, leg pain, headache, and palpitations which led to withdrawal from the study $(20,23)$. In randomized studies carried out on patients with a chronic obstructive pulmonary disease (COPD), the administration of oral NAC at a daily dose of 1,200 mg exerted an overall safety profile consistent with the established safety profile of $600 \mathrm{mg} /$ day NAC treatment $(24,25)$. In very high doses of oral NAC (1,800 mg/day), a slightly higher frequency of mild gastrointestinal symptoms was found in patients with COPD (gastrointestinal symptoms in 5/23 of NAC-treated patients vs 1/22 of placebo-treated patients) (26), and a higher occurence of cardiac complaints was observed in NAC-treated patients with an idiopathic pulmonary fibrosis (IPF) $(6.8 \%$ in NAC-treated vs $1.4 \%$ in placebo-treated patients) (27). However, a more recent study demonstrated that positive and adverse effects of NAC on patients with IPF depend on genotype characteristics (28). The occurence of adverse effects may be also related to the route of administration. For instance, in our experiments in rabbits with a meconium-induced acute lung injury, the intravenous administration of NAC at a dose of $10 \mathrm{mg} / \mathrm{kg}$ was associated with a short-term increase in blood pressure and changes in several parameters of the heart rate variability (29). Nevertheless, Meyer et al. compared the effects of three different doses $(600 \mathrm{mg}, 1,800 \mathrm{mg}$, and $4,800 \mathrm{mg}$ ) of intravenous NAC given to patients with IPF and healthy controls and found no differences in the occurence of adverse effects (30). Because of anticoagulant action and inhibition of platelet functions, the use of NAC should be considered in patients with bleeding disorders and anemia $(20,31)$.

Proposed mechanisms of action of NAC are listed in Table 1.

Table 1 Mechanisms of action of NAC (modified according to Ref. 5).

\section{Mechanisms of NAC action}

Direct scavenging of ROS

Restoration of GSH

Reduction of inflammation via suppression of NF-кB

Mucolytic action

Metal chelation

Stabilization of proteins
Protection and repair of DNA

Reduction of mitochondria apoptosis

Glutamate/dopamine homeostasis

Antiviral action

Inhibition of vascular permeability

Increase in ATP and NO production

Abbreviations: ATP: adenosine triphosphate, DNA: deoxyribonucleic acid, GSH: glutathione, NAC: $\mathrm{N}$-acetylcysteine, NF- B: nuclear factor kappa B, NO: nitric oxide, ROS: reactive oxygen species.

\section{Therapeutical use of NAC}

NAC has been clinically used for decades for the treatment of a wide variety of medical conditions (5), such as paracetamol overdose (32), metal-induced toxicity (33), and certain psychiatric disorders modulating glutamatergic, neurotropic, and inflammatory pathways (34). Animal studies showed that NAC can protect from oxidative stress and inflammation in many other conditions, such as a brain damage induced by transient cerebral ischemia (35), infection-induced pain and inflammation (36), or infiltration of thyroid gland by inflammatory cells in autoimmune thyroiditis (37). However, NAC may be of benefit in many 
respiratory diseases, such as COPD, chronic bronchitis, cystic fibrosis, or acute respiratory distress syndrome (ARDS) including COVID-19-induced ARDS (38, 39). In our experiments, the intravenous administration of NAC significantly reduced the markers of oxidative stress and inflammation in rabbits with the meconium-induced acute lung injury $(40,41)$, while an additional improvement was observed in combination with an intratracheal delivery of exogenous surfactant $(42,43)$.

\section{NAC in the lung silicosis \\ In vitro studies}

In murine macrophage cell line 264.7 exposed to silica, a pretreatment with NAC decreased silica-induced TNF $\alpha$ mRNA and protein levels and mRNA levels of macrophage inflammatory protein (MIP)-2, MIP-1 $\alpha$, MIP-1 $\beta$, and monocyte chemotactic protein (MCP)-1 (44). The authors concluded that the anti-inflammatory response to the administration of antioxidant NAC indicates a very close relationship between the silica-induced overproduction of ROS and the inflammation (44). This opinion was confirmed by other authors who found that silica exposure to rat2 fibroblast cell line caused a rapid degradation of peroxiredoxin, an important antioxidant system, and induced a degradation of $\mathrm{NF}-\kappa \mathrm{B}$ inhibitor IкB- $\alpha$. The pretreatment of the cells with $1 \mathrm{mM}$ NAC inhibited a suppression of IкB- $\alpha$ and blocked a silica-induced accumulation of ROS but had no effect on peroxiredoxins I and II (11). The silica-induced activation of $\mathrm{NF}-\kappa \mathrm{B}$ pathway leading to a two-fold increase in production of ROS was also demonstrated in murine peritoneal macrophage cell line RAW 264.7, while a pretreatment with 1 and $10 \mathrm{mM}$ NAC effectively reduced activation of NF- $\mathrm{KB}$ (45).

In AM isolated from silica-instilled rats, silica elevated intracellular ROS concentrations, resulting in a decrease in intracellular GSH and cysteine and a sustained presence of apoptotic AM which were characterized by a decreased mitochondrial transmembrane potential, an increased mitochondrial release of cytochrome $\mathrm{C}$, and activated caspase- 9 and caspase-3 compared to control group. Silica induced production of IL- $1 \beta$ and TNF $\alpha$ in AM which was inhibited by NAC. NAC also prevented intracellular GSH depletion but not apoptosis in AM (13). Similarly, in rat AM exposed in vitro to silica, the silica-induced apoptosis was accompanied with lowering of intracellular and mitochondrial GSH which was effectively blocked by NAC as well as the production of IL-1 $\beta$ and TNF $\alpha$ by AM, however, NAC did not protect the cells from apoptosis (46).

In rat AM NR8383 cells, the exposure to silica induced both inflammation and autophagy. The pretreatment with NAC decreased the expressions of pro-inflammatory cytokine TNF $\alpha$ and pro-fibrotic transforming growth factor- $\beta$ (TGF- $\beta$ ) but the absence of influencing PI3K/Akt/mTOR signaling autophagy pathway suggests that the anti-inflammatory response of NAC might be partially related to the inhibition of the release of lysosomal enzymes, the reduction of the stimulation of fibroblasts, eventually to the reduction of collagen and fibronectin synthesis by fibroblasts (47).

Of course, the value of the results obtained from in vitro studies is limited in comparison to the value of in vivo models or human studies. In in vitro measurements, the effects of NAC may be observed only in a sample of selected type of pulmonary cells without interactions with other lung cells or immune cells as it is possible in in vivo conditions. Based on these facts, it is impossible to transfer directly e.g. the dose of NAC used in in vitro study to in vivo experiments or to human study. Nevertheless, the measurements on isolated cell lines may bring valuable information on effects of the treatment agent on the cellular level, which may serve as a baseline for following in vivo experiments.

\section{In vivo studies}

The effects of NAC treatment have been tested almost exclusively on silica-injured rats or mice. Rats exposed to an intratracheal instillation of silica particles were treated with NAC at a dose of $500 \mathrm{mg} / \mathrm{kg}$ orally every day for 7 days before and up to 28 days after the silica administration. The treatment with NAC reduced the fibrotic score, decreased concentrations 
of hydroxyproline, a marker of collagen production, and malondialdehyde (MDA), a marker of oxidative stress, and prevented silica-induced increases in TNF $\alpha$, IL-8 and high-sensitivity C-reactive protein in the bronchoalveolar lavage fluid (BALF) and serum (48).

In other rat model of silicosis, the delivery of NAC by gavage at a dose of $600 \mathrm{mg}$ given since the silica instillation for up to 28 days, led to lighter histopathological changes in the lung with no cell nodules or silicotic nodules formation compared to the non-treated group. In addition, NAC reduced the contents of mitochondrial apoptosis pathway-related proteins cytochrome $\mathrm{C}$, caspases-3 and -9 , declined the contents of fibrosis markers procollagens-I and -III, decreased the intracellular content of ROS and prevented a decrease in mitochondrial transmembrane potential (MTP) (49).

In mice, NAC was given by gavage every day at three different doses: a low dose (1.73 $\mathrm{mg} / 20 \mathrm{~g})$, a moderate dose $(3.46 \mathrm{mg} / 20 \mathrm{~g})$, or at a high dose $(5.19 \mathrm{mg} / 20 \mathrm{~g})$, respectively, for $24 \mathrm{~h}$ or $1,2,3,4$, or 5 months to observe the effects of NAC on silica-induced inflammation and fibrosis. NAC reduced a generation of pro-inflammatory cytokines in BALF and decreased a level of lipoperoxidation marker MDA, with a stronger effect observed for moderate and high doses of NAC. On the other hand, NAC markedly enhanced the activities of antioxidant markers glutathione peroxidase and superoxide dismutase, and elevated the total antioxidant capacity. NAC treatment also down-regulated the oxidising enzymes NADPH oxidase 2, inducible nitric oxide synthase, superoxide dismutase 2, and xanthine oxidase. Moreover, NAC prevented a pulmonary injury including a fibrogenic response with a deposition of collagen. NAC also partially reversed an epithelial-mesenchymal transition (EMT) and a mesenchymal cell mobility, as confirmed by an increased expression of E-cadherin, which is down-regulated in fibrosis, decreased an expression of vimentin, a marker of mesenchymally-derived cells undergoing EMT, and lowered an expression of cytochrome C, which may be released from mitochondrial membrane in an injury and serves as a pro-inflammatory and pro-fibrotic factor (17).

We are aware of serious limitations which arise from the use of animal models of silicosis. The effects of the treatment may be influenced by inter-species differences (rat $v s$ human, mouse $v s$ human etc.) in the response to silica as well as to the given therapy because of the differences in immune responses, the specifics of inflammatory and metabolic pathways, etc. Therefore, the dose of the given treatment cannot be transfered directly from the animal model to humans. In addition, in animal models, silica is usually instilled into the trachea of animals homogenously to both lung lobes by a syringe, thus, also the mode of the lung affection is different from cumulative targeting the lung by inhalation in humans. An additional limitation is caused by the use of healthy young animals without any other concomittant diseases, which is a different situation from human disorder where serious lung silicosis is usually detected in older men where the lung silicosis is often associated with other chronic diseases, smoking, etc. Nevertheless, the use of animal models is the best way how to test at least in the laboratory conditions a potential of new treatment approaches before their introduction in the clinical studies. Pre-clinical testing reduces potential risks and represents a valuable pre-clinical base for clinical studies.

\section{Clinical studies}

After the publication of in vivo study where a combined therapy with NAC and tetrandrine in silicotic rats was more effective in anti-inflammatory, antioxidant and anti-fibrotic action than the treatments given separately (50), this combined treatment has started to be used also in the clinical studies (51-53). Tetrandrine isolated from the root of Stephania tetrandra S. Moore belongs to a group of bisbenzylisoquinoline alkaloids. Tetrandrine is used in traditional Chinese medicine because of its multiple anti-inflammatory effects including the reduction of ROS production, enhanced autophagic flux, the reversal of multi-drug resistance, influencing caspase pathway, cell cycle arrest and by modification of calcium channels. In silicosis, tetrandrine may alleviate pulmonary fibrosis and inflammation via reducing the level of type I and III collagen mRNA and collagen deposition in the lung $(54,55)$. 
<smiles>CC(=O)N[C@@H](CS)C(=O)O</smiles>

N-acetylcysteine $\left(\mathrm{C}_{5} \mathrm{H}_{9} \mathrm{NO}_{3} \mathrm{~S}\right)$

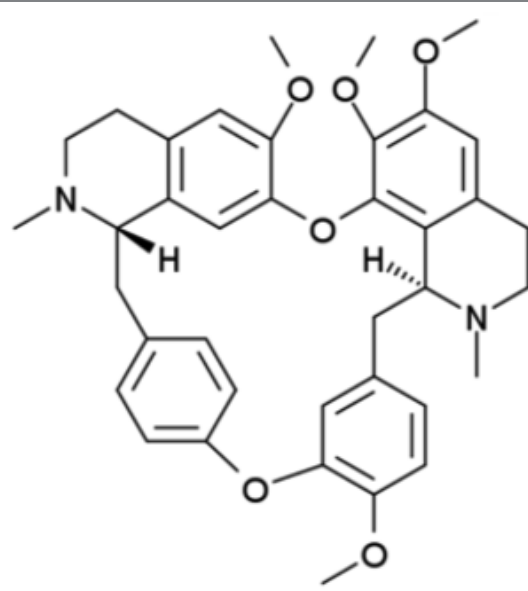

Tetrandrine $\left(\mathrm{C}_{38} \mathrm{H}_{42} \mathrm{~N}_{2} \mathrm{O}_{6}\right)$
Fig. 1. Chemical structures of $\mathrm{N}$-acetylcysteine and tetrandrine

In a group of 88 patients with silicosis, tetrandrine at a dose of $60 \mathrm{mg}-100 \mathrm{mg} / \mathrm{time}$ was given 3 times a day for 6 days and then stopped for 1 day, while a course of tetrandrine treatment lasted for 3 months. This treatment was combined with a delivery of NAC effervescent tablets of $600 \mathrm{mg}$ at a dose of 1 tablet/time, 1-2 times/day, which lasted for 8 months. The combined treatment decreased a respiratory rate, enhanced forced vital capacity (FVC) and first second forced expiratory volume (FEV1), and decreased plasma levels of TNF $\alpha$ and IL-6 compared to the control group (51).

Similar positive results of the combined NAC+tetrandrine treatment were observed in the study by Guo et al. (52) where the treatment was given to 132 patients with lung silicosis in the following regime: NAC effervescent tablets $600 \mathrm{mg} /$ time, 2 times daily; tetrandrine tablets $100 \mathrm{mg} /$ time, 2 times daily; while the drug delivery was stopped after 6 days for 1 day; and the treatment was given in 4 courses for 8 months. The treatment combination improved the pulmonary function tests, an exercise tolerance of patients demonstrated by a 6 min walk test, and an occurance of symptoms such as chest tightness, chest pain, cough, or sputum production (52).

In a group of 79 patients with silicosis, a combined treatment was given as following: NAC effervescent tablets of $600 \mathrm{mg}$ were administered twice a day, with 12 days long duration of the treatment course. The patients received one course per month in the first two months, then one course every 2 months, and the patients received 4 courses in total. Tetrandrine tablets of 60-100 $\mathrm{mg}$ were given three times a day for 6 days a week. One course of the treatment lasted 3 months, then stopped for 1 month, and subsequently continued in the second course for 3 months. This design of the treatment improved the rates of respiratory distress, chest pain, and dyspnea compared to the control group and increased an exercise tolerance expressed by a 6-min walk test. In addition, NAC+tetrandrine treatment reduced the plasma levels of TGF- $\beta 1$, a potent fibrogenic and pro-inflammatory factor, and decreased matrix metalloproteinase (MMP)-7, a key factor in fibrosis which degrades cytoplasmic matrix components (53).

\section{CONCLUSIONS}

Overexpression of ROS is a fundamental pathomechanism responsible for origin and chronic persistence of changes in pulmonary silicosis. Recent studies have demonstrated that the administration of antioxidants, e.g. of NAC, may not only decrease the oxidative 
stress but may also alleviate the lung inflammation and fibrosis. In additon, the results from animal studies demonstrating the benefits of preventive use of NAC indicate that regarding a long-term development of lung silicosis the preventive use of NAC or other antioxidants may mitigate the extent of silica-induced changes and, therefore, the longterm use of antioxidants in silicosis-predisposed occupations is one of the treatment possibilities. Nevertheless, we are aware that additional clinical studies are necessary to evaluate the positive but also the possible adverse effects before any recommendations about the use of NAC for the treatment of silicosis could be made. However, the first clinical studies from China indicate that the use of NAC may be one of valuable approaches for diminishing the lung injury and improving the prognosis of patients with pulmonary silicosis.

\section{Acknowledgements:}

This work was supported by the projects APVV-15-0075, VEGA 1/0356/18 and VEGA $1 / 0131 / 22$.

\section{REFERENCES}

1. Greenberg MI, Waksman J, Curtis J. Silicosis: a review. Dis Mon 2007; 53 (8): 394-416.

2. Pollard KM. Silica, Silicosis, and Autoimmunity. Front Immunol 2016; 7: 97.

3. Barnes H, Goh NSL, Leong TL, Hoy R. Silica associated lung disease: An old world exposure in modern industries. Respirology 2019; 24 (12): 1165-75.

4. Adamcakova J, Mokra D. New Insights into Pathomechanisms and Treatment Possibilities for Lung Silicosis. Int J Mol Sci 2021; 22 (8): 4162.

5. Schwalfenberg GK. N-Acetylcysteine: A Review of Clinical Usefulness (an Old Drug with New Tricks). J Nutr Metab 2021; 2021: 9949453.

6. Vallyathan V, Castranova V, Pack D, Leonard S, Shumaker J, Hubbs AF, Shoemaker DA, Ramsey DM, Pretty JR, McLaurin JL, et al. Freshly fractured quartz inhalation leads to enhanced lung injury and inflammation. Potential role of free radicals. Am J Respir Crit Care Med 1995; 152 (3): 1003-9.

7. Hamilton RF Jr, Thakur SA, Holian A. Silica binding and toxicity in alveolar macrophages. Free Radic Biol Med 2008; 44 (7): 1246-58.

8. Porter DW, Ye J, Ma J, Barger M, Robinson VA, Ramsey D, McLaurin J, Khan A, Landsittel D, Teass A, Castranova V. Time course of pulmonary response of rats to inhalation of crystalline silica: NF-kappa B activation, inflammation, cytokine production, and damage. Inhal Toxicol 2002; 14 (4): 349-67.

9. Morgan MJ, Liu ZG. Crosstalk of reactive oxygen species and NF- B signaling. Cell Res 2011; 21 (1): 103-15.

10. Harijith A, Ebenezer DL, Natarajan V. Reactive oxygen species at the crossroads of inflammasome and inflammation. Front Physiol 2014; 5: 352.

11. Seo MS, Kim JK, Lim Y, Kang SW, Cho YJ, Lee WK, Kim HJ, Cho KK, Lee KH, Rhee SG. Rapid degradation of PrxI and PrxII induced by silica in Rat2 cells. Biochem Biophys Res Commun 1999; 265 (2): 541-4.

12. Biswas R, Bunderson-Schelvan M, Holian A. Potential role of the inflammasome-derived inflammatory cytokines in pulmonary fibrosis. Pulm Med 2011; 2011: 105707.

13. Hu S, Zhao H, Al-Humadi NH, Yin XJ, Ma JK. Silica-induced apoptosis in alveolar macrophages: evidence of in vivo thiol depletion and the activation of mitochondrial pathway. $J$ Toxicol Environ Health A 2006; 69 (13): 1261-84.

14. Kim KH, Lee MS. Autophagy--a key player in cellular and body metabolism. Nat Rev Endocrinol 2014; 10 (6): 322-37.

15. Zhao H, Wang Y, Qiu T, Liu W, Yao P. Autophagy, an important therapeutic target for pulmonary fibrosis diseases. Clin Chim Acta 2020; 502: 139-47. 
16. Adamcakova J, Mokra D. Herbal compounds in the treatment of pulmonary silicosis. Physiol Res 2021; 70 (Suppl 1): xxx (accepted for publication).

17. Huang H, Chen M, Liu F, Wu H, Wang J, Chen J, Liu M, Li X. N-acetylcysteine therapeutically protects against pulmonary fibrosis in a mouse model of silicosis. Biosci Rep 2019; 39: BSR20190681.

18. Aldini G, Altomare A, Baron G, Vistoli G, Carini M, Borsani L, Sergio F. N-Acetylcysteine as an antioxidant and disulphide breaking agent: the reasons why. Free Radic Res 2018; 52 (7): 75162.

19. Tenório MCDS, Graciliano NG, Moura FA, Oliveira ACM, Goulart MOF. N-Acetylcysteine (NAC): Impacts on Human Health. Antioxidants (Basel) 2021; 10 (6): 967.

20. Calverley P, Rogliani P, Papi A. Safety of N-Acetylcysteine at High Doses in Chronic Respiratory Diseases: A Review. Drug Saf 2021; 44 (3): 273-90.

21. Stey C, Steurer J, Bachmann S, Medici TC, Tramèr MR. The effect of oral N-acetylcysteine in chronic bronchitis: a quantitative systematic review. Eur Respir J 2000; 16 (2): 253-62.

22. Grandjean EM, Berthet P, Ruffmann R, Leuenberger P. Efficacy of oral long-term N-acetylcysteine in chronic bronchopulmonary disease: a meta-analysis of published double-blind, placebocontrolled clinical trials. Clin Ther 2000; 22 (2): 209-21.

23. Hansen NC, Skriver A, Brorsen-Riis L, Balsløv S, Evald T, Maltbaek N, Gunnersen G, Garsdal P, Sander P, Pedersen JZ, et al. Orally administered N-acetylcysteine may improve general wellbeing in patients with mild chronic bronchitis. Respir Med 1994; 88 (7): 531-5.

24. Zheng JP, Wen FQ, Bai CX, Wan HY, Kang J, Chen P, Yao WZ, Ma LJ, Li X, Raiteri L, Sardina M, Gao Y, Wang BS, Zhong NS; PANTHEON study group. Twice daily N-acetylcysteine $600 \mathrm{mg}$ for exacerbations of chronic obstructive pulmonary disease (PANTHEON): a randomized, doubleblind placebo-controlled trial. Lancet Respir Med 2014; 2 (3): 187-94.

25. Tse HN, Raiteri L, Wong KY, Yee KS, Ng LY, Wai KY, Loo CK, Chan MH. High-dose N-acetylcysteine in stable COPD: the 1-year, double-blind, randomized, placebo-controlled HIACE study. Chest 2013; 144 (1): 106-18.

26. Johnson K, McEvoy CE, Naqvi S, Wendt C, Reilkoff RA, Kunisaki KM, Wetherbee EE, Nelson D, Tirouvanziam R, Niewoehner DE. High-dose oral N-acetylcysteine fails to improve respiratory health status in patients with chronic obstructive pulmonary disease and chronic bronchitis: a randomized, placebo-controlled trial. Int J Chron Obstruct Pulmon Dis 2016; 11: 799-807.

27. Martinez FJ, de Andrade JA, Anstrom KJ, King TE Jr, Raghu G; Idiopathic Pulmonary Fibrosis Clinical Research Network. Randomized trial of acetylcysteine in idiopathic pulmonary fibrosis. N Engl J Med 2014; 370 (22): 2093-101.

28. Oldham JM, Ma SF, Martinez FJ, Anstrom KJ, Raghu G, Schwartz DA, Valenzi E, Witt L, Lee C, Vij R, Huang Y, Strek ME, Noth I; IPFnet Investigators. TOLLIP, MUC5B, and the Response to NAcetylcysteine among Individuals with Idiopathic Pulmonary Fibrosis. Am J Respir Crit Care Med 2015; 192 (12): 1475-82.

29. Mokra D, Tonhajzerova I, Pistekova H, Visnovcova Z, Drgova A, Mokry J, Calkovska A. Cardiovascular effects of N-acetylcysteine in meconium-induced acute lung injury. Adv Exp Med Biol 2015; 832: 35-43.

30. Meyer A, Buhl R, Kampf S, Magnussen H. Intravenous N-acetylcysteine and lung glutathione of patients with pulmonary fibrosis and normals. Am J Respir Crit Care Med 1995; 152 (3): 105560.

31. Niemi TT, Munsterhjelm E, Pöyhiä R, Hynninen MS, Salmenperä MT. The effect of N-acetylcysteine on blood coagulation and platelet function in patients undergoing open repair of abdominal aortic aneurysm. Blood Coagul Fibrinolysis 2006; 17 (1): 29-34.

32. Chiew AL, Gluud C, Brok J, Buckley NA. Interventions for paracetamol (acetaminophen) overdose. Cochrane Database Syst Rev 2018; 2 (2): CD003328.

33. Yeh IJ, Wang TY, Lin JC, Lin TJ, Chang JS, Yen MC, Liu YH, Wu PL, Chen FW, Shih YL, Peng CY. Optimal Regimen of N-Acetylcysteine on Chromium-Induced Renal Cell Damage. Metabolites 2019; 9 (9):172. 
34. Ooi SL, Green R, Pak SC. N-Acetylcysteine for the Treatment of Psychiatric Disorders: A Review of Current Evidence. Biomed Res Int 2018; 2018: 2469486.

35. Cuzzocrea S, Mazzon E, Costantino G, Serraino I, Dugo L, Calabrò G, Cucinotta G, De Sarro A, Caputi AP. Beneficial effects of n-acetylcysteine on ischaemic brain injury. Br J Pharmacol 2000; 130 (6): 1219-26.

36. Crupi R, Gugliandolo E, Siracusa R, Impellizzeri D, Cordaro M, Di Paola R, Britti D, Cuzzocrea S. $\mathrm{N}$-acetyl-L-cysteine reduces Leishmania amazonensis-induced inflammation in BALB/c mice. BMC Vet Res 2020; 16 (1): 13.

37. Poncin S, Colin IM, Decallonne B, Clinckspooor I, Many MC, Denef JF, Gérard AC. N-acetylcysteine and 15 deoxy-\{delta\}12,14-prostaglandin J2 exert a protective effect against autoimmune thyroid destruction in vivo but not against interleukin-1\{alpha\}/interferon \{gamma\}-induced inhibitory effects in thyrocytes in vitro. Am J Pathol 2010; 177 (1): 219-28.

38. Jorge-Aarón RM, Rosa-Ester MP. N-acetylcysteine as a potential treatment for COVID-19. Future Microbiol 2020; 15: 959-62.

39. Poe FL, Corn J. N-Acetylcysteine: A potential therapeutic agent for SARS-CoV-2. Med Hypotheses 2020; 143: 109862.

40. Mokra D, Drgova A, Mokry J, Antosova M, Durdik P, Calkovska A. N-acetylcysteine effectively diminished meconium-induced oxidative stress in adult rabbits. J Physiol Pharmacol 2015; 66 (1): 101-10.

41. Mokra D, Drgova A, Petras M, Mokry J, Antosova M, Calkovska A. N-acetylcysteine alleviates the meconium-induced acute lung injury. Adv Exp Med Biol 2015; 832: 59-67.

42. Kopincová J, Mokrá D, Mikolka P, Kolomazník M, Čalkovská A. N-acetylcysteine advancement of surfactant therapy in experimental meconium aspiration syndrome: possible mechanisms. Physiol Res 2014; 63 (Suppl 4): S629-42.

43. Mikolka P, Kopincova J, Mikusiakova LT, Kosutova P, Calkovska A, Mokra D. Antiinflammatory Effect of N-Acetylcysteine Combined with Exogenous Surfactant in Meconium-Induced Lung Injury. Adv Exp Med Biol 2016; 934: 63-75.

44. Barrett EG, Johnston C, Oberdörster G, Finkelstein JN. Antioxidant treatment attenuates cytokine and chemokine levels in murine macrophages following silica exposure. Toxicol Appl Pharmacol 1999; 158 (3): 211-20.

45. Kang JL, Go YH, Hur KC, Castranova V. Silica-induced nuclear factor-kappaB activation: involvement of reactive oxygen species and protein tyrosine kinase activation. J Toxicol Environ Health A 2000; 60 (1): 27-46.

46. Hu S, Zhao H, Yin XJ, Ma JK. Role of mitochondria in silica-induced apoptosis of alveolar macrophages: inhibition of apoptosis by rhodamine $6 \mathrm{G}$ and N-acetyl-L-cysteine. J Toxicol Environ Health A 2007; 70 (17): 1403-15.

47. Li N, Shi F, Wang X, Yang P, Sun K, Zhang L, Hao X, Li X, Li J, Jin Y. Silica dust exposure induces pulmonary fibrosis through autophagy signaling. Environ Toxicol 2021; 36 (7): 1269-77.

48. Zhang H, Yin G, Jiang H, Zhang C. High-dose N-acetylcysteine decreases silica-induced lung fibrosis in the rat. J Int Med Res 2013; 41 (4): 1179-86.

49. Zhang L, He YL, Li QZ, Hao XH, Zhang ZF, Yuan JX, Bai YP, Jin YL, Liu N, Chen G, Yun X, Yao SQ. N-acetylcysteine alleviated silica-induced lung fibrosis in rats by down-regulation of ROS and mitochondrial apoptosis signaling. Toxicol Mech Methods 2014; 24 (3): 212-9.

50. Xiao Y, Xia H, Zhu L, Li X, Chen R, Yin X, Jiang Z, Feng L, Chen J, Yu M, Lou J, Zhang X. Study on the therapeutic effects of tetrandrine combined with $\mathrm{N}$-acetylcysteine on experimental silicosis of rats (article in Chinese). Zhonghua Lao Dong Wei Sheng Zhi Ye Bing Za Zhi 2015; 33 (7): 51922.

51. Sun J, Song P, Wang Y, Chen Y. Clinical efficacy of acetylcysteine combined with tetrandrine tablets in the treatment of silicosis and the effect on serum IL-6 and TNF-alpha. Exp Ther Med 2019; 18 (5): 3383-8.

52. Guo X, Qi J, Li H, Xing Z. Clinical efficacy of acetylcysteine combined with tetrandrine tablets on patients with silicosis and its effect on exercise tolerance and pulmonary function. Exp Ther Med 2020; 20 (2): 1285-90. 
53. Zhang J, Wang Y, Zhang S, Li J, Fang H. Effects of tetrandrine combined with acetylcysteine on exercise tolerance, pulmonary function and serum TNF- $\beta 1$ and MMP-7 in silicosis patients. Exp Ther Med 2020; 19 (3): 2195-201.

54. Xie QM, Tang HF, Chen JQ, Bian RL. Pharmacological actions of tetrandrine in inflammatory pulmonary diseases. Acta Pharmacol Sin 2002; 23 (12): 1107-13.

55. Bhagya N, Chandrashekar KR. Tetrandrine--A molecule of wide bioactivity. Phytochemistry 2016; 125: 5-13.

Received: October, 29, 2021

Accepted:November, 29, 2021 